\title{
Blade Element Momentum Theory for a Tidal Turbine
}

\author{
C. R. Vogel ${ }^{\mathrm{a}, *}$, R. H. J. Willden ${ }^{\mathrm{a}}$, G. T. Houlsby ${ }^{\mathrm{a}}$ \\ ${ }^{a}$ Department of Engineering Science, University of Oxford, OX1 3PJ, United Kingdom
}

\begin{abstract}
A key hydrodynamic difference between tidal current and wind turbines is the volume-flux constrained flow field in which tidal turbines operate and the resulting streamwise static pressure difference that develops in the flow passage. Blade Element Momentum (BEM) theory is extended to account analytically for the effects of blockage and the development of the static pressure difference in the flow passage and shows agreement in thrust and power predictions to within $\pm 3 \%$ of equivalent blade resolved simulations. The confined flow BEM model is employed to study two different power capping strategies: varying the rotational speed with fixed pitch blades; and pitching the blades to feather at constant rotational speed. Pitch-to-feather achieves reduced thrust above rated flow speed which leads to a greater extractable resource than achievable with overspeed control, due to the feedback between device thrust and available tidal resource. Flow confinement is shown to reduce the flow speed at which rated power occurs, and increases the rotor loads and power below rated conditions. It is also shown that root bending moments, which affect fatigue damage rates, increase with flow confinement.
\end{abstract}

Keywords: Tidal stream turbines, Power capping, Blockage effect, Blade element momentum theory

The adaptation of established techniques for the analysis of wind turbines has allowed much progress to be made in the understanding of axial flow tidal current turbines. The analysis of an actuator disc in a confined flow field by Garrett and Cummins established the importance of the blockage ratio, $B$, the ratio of rotor swept area to the cross sectional area of the surrounding flow passage [9]. It was shown that the maximum power coefficient of an actuator disc increases by a factor of $(1-B)^{-2}$ above the Lanchester-Betz limit of 0.593 when blockage effects are considered, allowing tidal current turbines to achieve higher power coefficients than wind turbines. Physically, this is because a static pressure drop is established in the blocked flow between far upstream and downstream of the turbine, allowing a greater pressure drop to be supported across the turbines, thereby increasing the peak thrust and extractable power as the local blockage ratio increases. Whereas the maximum extractable power for a wind turbine is achieved by reducing the flow speed through the turbine plane to two thirds of its upstream value, for a tidal turbine maximum power occurs at a monotonically reducing flow speed through the turbine plane as the blockage ratio is increased. Furthermore, as the blockage ratio increases and the bypass flow passage becomes more constrained, the flow speed bypassing the turbine increases [27].

There has been interest in the fields of both the wind and tidal stream energy in understanding and quantifying the effects of flow confinement on rotor performance, such as those encountered in wind tunnels and water flumes. Experimental investigations of tidal rotors have confirmed the uplift in rotor thrust and power that occurs with increasing blockage ratio [1], and Chamorro et al. [6] demonstrated that blockage effects can increase spanwise flows along rotor blades and increase the level of rotor-induced turbulence in the wake. Similar dependence on blockage has been observed for wind turbines in tunnels, with Sarlak et al. [19] noting that the significance of the effects also depends on rotor thrust. Understanding the effect of blockage on rotor performance has particular importance for rotor design, as increased blade loading can result in larger

\footnotetext{
* Corresponding author

Email address: christopher.vogel@eng.ox.ac.uk (C. R. Vogel)
} 
root bending moments [18], as well as allowing the for possibility of improved device performance if interturbine spacing is carefully controlled to exploit constructive interference effects between turbines through flow confinement $[2,26]$.

The change in flow conditions due to blockage effects can be related to turbine performance, given rotor properties such as aerofoil lift and drag coefficients, and blade twist and solidity using Blade Element Momentum (BEM) theory. BEM theory has been widely used for wind turbine analysis and design, and has increasingly been applied to tidal current turbine analysis and design. BEM theory, as applied to wind turbines, does not consider the effect of blockage on rotor performance. One approach for addressing this in the context of tidal turbines has been to embed the blade element analysis within a computational fluid dynamics (CFD) simulation, where the blade element model is unchanged from that for wind turbines and the effect of the constrained flow field is simulated explicitly to account for blockage effects (for examples, see [13], [20], and [7]). An alternative to performing blade element-CFD simulations is to modify the momentum equations in BEM theory to take the effects of blockage into account, such as by using the models presented in [9] or [28]. A blockage-corrected analytic BEM model provides a tractable tool which may be used in the design and analysis of tidal turbines. In particular, a blockage-corrected BEM tool provides an efficient method for investigating the multitude of design cases necessary to develop a robust rotor design, such as rotor performance in off-design conditions, performance during power capped operation, and rotor design in blocked conditions. Turbine design in blocked conditions is particularly important if designs are to approach the higher power coefficients predicted by the simple analytic theory of Garrett and Cummins.

This paper develops a blockage-corrected BEM model using the rigid lid momentum model of Garrett and Cummins to provide a semi-analytic tool which is validated against blade boundary layer resolved simulations. The blockage-corrected tool allows the rapid exploration of the blockage and tip speed ratio operational space of turbines, and is used to investigate the performance of rotors in blocked conditions. The rotor characteristics (performance as a function of flow speed) determined with such an analytic BEM method allow long turbine fences to be simulated without the need for expensive three dimensional numerical models by providing sub-grid scale turbine models suitable for depth-averaged simulations.

\section{Effect of Blockage on Momentum Theory}

Wind turbines are generally modelled as operating in unconfined flows in which there is full recovery of the static pressure far downstream of the rotor. Garrett and Cummins showed with Linear Momentum Actuator Disc Theory (LMADT) that momentum removal from a constant volume-flux flow by a turbine results in a static pressure deficit in the far wake of the rotor, and hence a static pressure gradient is established in the flow passage containing the turbine [9]. This static pressure gradient results in a greater achievable pressure difference across the rotor plane as compared to unblocked flow. There is hence a greater flow speed through the rotor plane of the blocked rotor for a given level of thrust, which generally increases the torque and therefore the power, depending on the angle of attack of the blades.

The flow speed through the rotor plane and in the wake of the rotor depends on the applied thrust and blockage ratio, and consequently the momentum equations within the unconfined BEM formulation must be modified to account for this additional dependency. The changes to momentum theory for the confined flow case are outlined herein; a detailed account of unconfined BEM theory may be found in [5].

Figure 1 is a schematic of the flow past a rotor in a volume-flux constrained flow. The upstream boundary of the confined BEM model is derived from the larger-scale flow around the turbine, which may, for example, be the flow through a fence of turbines that is being simulated in a depth-averaged coastal scale model. This provides the upstream flow speed, $u_{a}$, which is taken as the reference velocity for the turbine-scale model. The flow through the rotor swept area is divided into $N$ independent annular streamtubes of width $\delta r$, which are bounded by the bypass flow. The downstream boundary of the finite blockage BEM model is the streamwise point at which the static pressure equalises across the annular streamtubes and the bypass flow, although the possibility of differing flow speeds between the bypass and each annular streamtube is allowed. 

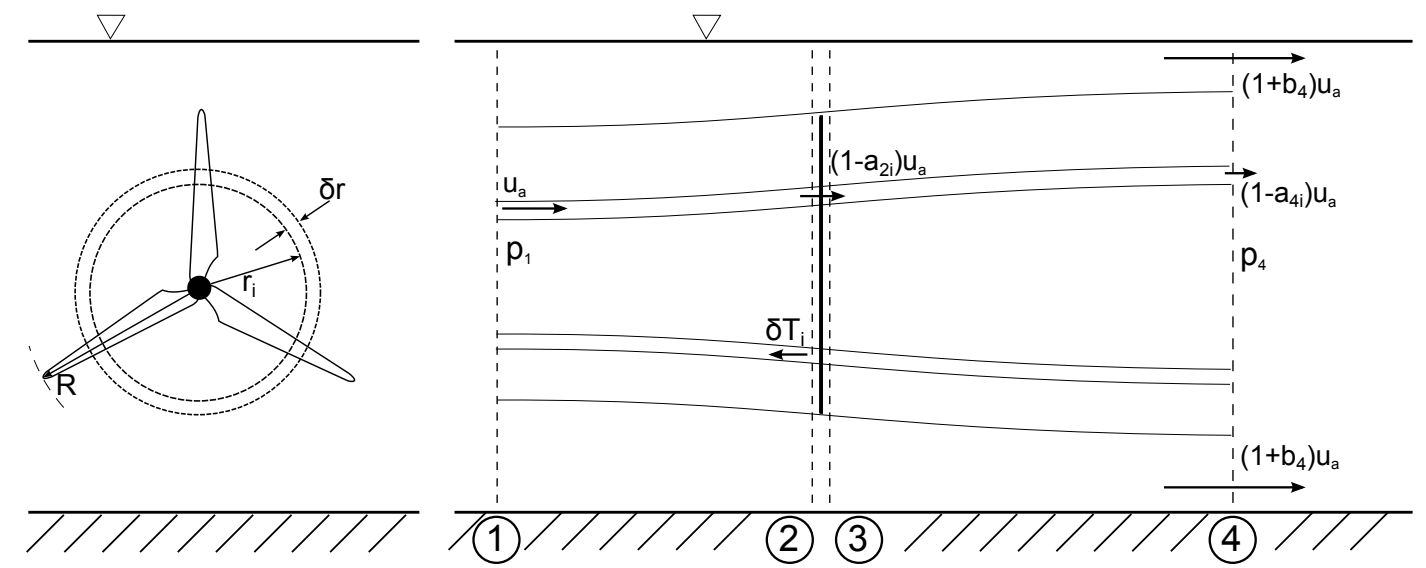

Figure 1: Diagram of the blockage corrected BEM model. Station one is far upstream of the rotor plane, station two just upstream of the rotor plane, and station four is far downstream of the rotor plane, where the static pressure equalises across the core and bypass flows.

The flow past the rotor is analysed at four stations; station one, which is far upstream of the rotor and unaffected by its operation (at least within this turbine-scale model), station two, which is immediately upstream of the rotor, station three, which is immediately downstream of the rotor, and station four, which is far downstream of the rotor at the position where static pressure may be considered as having equalised between the core and bypass streamtubes. The mixing processes between the core and bypass flows, which result in further energy removal from the flow, are assumed to occur entirely downstream of station four, and result in a uniform flow with speed identical to that far-upstream, as shown by Garrett and Cummins [9]. Energy extraction from a sub-critical open channel flow implies that the free surface elevation must decrease slightly far downstream. This phenomenon is negligible for the range of Froude numbers (0.10 to 0.20) at sites of interest for tidal stream energy, although could be incorporated into the analysis using computational models or momentum theory that includes free surface deformation, e.g. [11]. Assuming no other work is done on the fluid, momentum removal by the rotor results in a static pressure drop between stations one and four of $\Delta p=p_{1}-p_{4}$. The induction factors used to describe the velocity $u_{2 d i}$ through the $i^{\text {th }}$ streamtube at the rotor plane, $a_{2 i}$, and the velocity $u_{4 d i}$ in the wake of the turbine, $a_{4 i}$, are defined relative to the reference flow speed $u_{a}$ :

$$
u_{2 d i}=\left(1-a_{2 i}\right) u_{a} ; \quad u_{4 d i}=\left(1-a_{4 i}\right) u_{a} .
$$

Likewise, the induction factor, $b_{4}$, for the bypass velocity at the static pressure equalisation point, $u_{4 b}$, is defined relative to the reference velocity as:

$$
u_{4 b}=\left(1+b_{4}\right) u_{a} .
$$

Conservation of mass, momentum, and energy is considered in each of the annular streamtubes. At the rotor plane the $i^{\text {th }}$ annular streamtube is at a radius $r_{i}$ from the centre of rotation and has a radial thickness of $\delta r_{i}$ (usually assumed to be uniform across the blade span), so that $\delta A_{i} \approx 2 \pi r_{i} \delta r_{i}$. Conservation of mass requires:

$$
\delta A_{1 i} u_{a}=\delta A_{i} u_{a}\left(1-a_{2 i}\right)=\delta A_{4 i} u_{a}\left(1-a_{4 i}\right),
$$

where $\delta A_{1 i}$ and $\delta A_{4 i}$ are the cross-sectional areas of the $i^{\text {th }}$ streamtube at stations one and four respectively. Conservation of energy is described upstream and downstream of the rotor plane and through the rotor bypass by the Bernoulli equation:

$$
p_{1}-p_{2 i}=\frac{1}{2} \rho u_{a}^{2}\left(a_{2 i}^{2}-2 a_{2 i}\right), \quad p_{3 i}-p_{4}=\frac{1}{2} \rho u_{a}^{2}\left(a_{4 i}^{2}+2\left(a_{2 i}-a_{4 i}\right)-a_{2 i}^{2}\right), \quad p_{1}-p_{4}=\frac{1}{2} \rho u_{a}^{2}\left(b_{4}^{2}+2 b_{4}\right) .
$$

Conservation of momentum in the $i^{\text {th }}$ streamtube relates the thrust to the change in static pressure and momentum of the fluid:

$$
p_{1} \delta A_{1 i}+p^{*} \delta A^{*}-p_{4} \delta A_{4 i}-\delta T_{i}=-\rho \delta A_{i} u_{a}^{2}\left(1-a_{2 i}\right) a_{4 i},
$$


where $p^{*} \delta A^{*}$ is the unknown streamwise force acting on the surface of streamtube as it expands between stations one and four. Following Nishino and Willden [17], we assume slow expansion of the streamtube so that the unknown streamwise force may be accounted for with the approximation:

$$
p_{1} \delta A_{1 i}+p^{*} \delta A^{*}=p_{1} \delta A_{i}\left(1-a_{2 i}\right)+p^{*} \delta A^{*} \approx p_{1} \delta A_{4 i}=p_{1} \delta A_{i} \frac{\left(1-a_{2 i}\right)}{\left(1-a_{4 i}\right)},
$$

i.e., the additional force contribution from the expanding streamtube is approximated as the increase in force that would result from the static pressure at station one being applied over the streamtube cross-sectional area at station four. Equations (3) to (5) are combined in the usual way as described in [9] to yield the following key results used in the following analysis. Using conservation of mass, the momentum equation (5) can be manipulated to yield the incremental thrust $\delta T_{i}$ :

$$
\delta T_{i}=\rho \pi r_{i} u_{a}^{2} \frac{\left(1-a_{2 i}\right)}{\left(1-a_{4 i}\right)}\left(b_{4}^{2}+2\left(b_{4}+a_{4 i}\right)-2 a_{4 i}^{2}\right) \delta r_{i}
$$

The expressions in (4), combined with the definition that the incremental thrust is the product of the static pressure difference between stations two and three and the swept area of the annulus, $\delta T_{i}=\left(p_{2 i}-p_{3 i}\right) \delta A_{i}$, gives an expression for $\delta T_{i}$ in terms of $a_{4 i}$ and $b_{4}$ :

$$
\delta T_{i}=\rho \pi r_{i} u_{a}^{2}\left(b_{4}^{2}+2\left(a_{4 i}+b_{4}\right)-a_{4 i}^{2}\right) \delta r_{i}
$$

Equating the two expressions for annular thrust, (7) and (8), a cubic in $a_{4 i}$ is found:

$$
a_{4 i}^{3}-a_{4 i}^{2}\left(1+2 a_{2 i}\right)+a_{4 i}\left(2 a_{2 i}-\left(b_{4}^{2}+2 b_{4}\right)\right)+\left(b_{4}^{2}+2 b_{4}\right) a_{2 i}=0 .
$$

In the limit as $b_{4} \rightarrow 0$, which implies that the bypass flow speed tends to the free-stream flow speed, the unconfined wind turbine theory result of $a_{4 i}=2 a_{2 i}$ is recovered, and the familiar unconfined BEM model is recovered. Empirical relationships (e.g. the turbulent wake state model of Buhl [4]) between rotor thrust and induction factor are typically adopted when the thrust coefficient approaches unity, where unconfined momentum theory implies that nonphysical wake velocities develop. Similar empirical models have yet to be developed for confined flows, although it is expected that the transition to turbulent wake state modelling will occur at higher thrust coefficients as the wake flow speed increases with blockage for a given thrust level. Therefore, it is expected that confined momentum theory will produce physically meaningful wake velocities at higher thrust levels as the blockage ratio increases. However, the influence of blockage on turbulent wake state modelling represents an area of future work required to further advance analytic models of turbines in confined flows.

Closure of the system of equations for the confined BEM model requires an additional equation for $b_{4}$, which may be found through analysis of the bypass flow. Conservation of mass in the flow passage between stations one and four requires:

$$
-A_{c} b_{4}=\sum_{i=1}^{N} \delta A_{4 i}\left(1-a_{4 i}\right)-\sum_{i=1}^{N} \delta A_{4 i}\left(1+b_{4}\right),
$$

where the summation occurs over the $N$ annular streamtubes, and $A_{c}$ is the flow passage cross-sectional area. Equation (3) allows the streamtube cross-sectional area at station four to be written in terms of the streamtube area at the rotor plane and the ratio of induction factors at the rotor plane and at station four:

$$
-A_{c} b_{4}=\sum_{i=1}^{N} \delta A_{i} \frac{\left(1-a_{2 i}\right)}{\left(1-a_{4 i}\right)}\left(1-a_{4 i}\right)-\sum_{i=1}^{N} \delta A_{i} \frac{\left(1-a_{2 i}\right)}{\left(1-a_{4 i}\right)}\left(1+b_{4}\right) .
$$

Streamtube expansion downstream of the rotor is constrained by the flow passage boundaries, and is hence a function of the blockage ratio. Observation of numerical simulations (see Appendix A) shows that the streamtube ratio $\left(1-a_{2 i}\right) /\left(1-a_{4 i}\right)$ is, to a first approximation, uniform across the streamtubes, indicating that the streamtubes expand uniformly in the rotor wake. The exception to this occurs near the rotor tip, 
where the tip loss has a significant influence on the flow expansion in the numerical simulation. Hence, the streamtube expansion ratio is assumed to be constant in this model and defined as $\kappa=\left(1-a_{2 i}\right) /\left(1-a_{4 i}\right)$. The value of $\kappa \geq 1$ for physically meaningful turbine results, where $\kappa=2$ corresponds to the unconstrained wake expansion case of a wind turbine operating at the Betz limit. Furthermore, we define the average wake induction factor, $\overline{a_{4}}$, as an area-weighted average of the wake induction factors:

$$
\overline{a_{4}}=\frac{\sum_{i=1}^{N} \delta A_{4 i} a_{4 i}}{\delta A_{4 i}}=\frac{\sum_{i=1}^{N} \delta A_{i} \kappa a_{4 i}}{\sum_{i=1}^{N} \delta A_{i} \kappa}=\frac{1}{A_{d}} \sum_{i=1}^{N} \delta A_{i} a_{4 i}
$$

Noting that the swept area of the rotor, $A_{d}$, is defined as $A_{d}=\sum_{i=1}^{N} \delta A_{i}$, so that the blockage ratio $B=A_{d} / A_{c}$, Equation (11) becomes:

$$
b_{4}=B \kappa\left(\overline{a_{4}}+b_{4}\right) .
$$

The expansion of the wake, and hence the wake induction factor, is a consequence of the rotor thrust and constraining effect of the flow passage boundaries. Expressions for $\kappa$ and $\overline{a_{4}}$ may be found by considering the momentum removed by an equivalent single actuator disk applying the same total thrust as the actuator disk, divided into concentric annuli, analysed herein. Following the actuator disk model of Garrett and Cummins [9], continuity allows $\kappa$ to be written as:

$$
\kappa=\frac{b_{4}-\overline{a_{4}}+2}{b_{4}-2 \overline{a_{4}}-1} .
$$

Equations (13) and (14) are used to determine $\overline{a_{4}}$, which in combination with Equation (8) allows an expression for $b_{4}$ in terms of the rotor thrust coefficient, $C_{T}=T / \frac{1}{2} \rho A_{d} u_{a}^{2}$, where $T=\sum \delta T_{i}$ is the total thrust applied by the rotor, and the blockage ratio $B$ to be derived:

$$
3\left(1+b_{4}\right)^{4}-4\left(1+b_{4}\right)^{3}-2\left(1+b_{4}\right)^{2}\left(1+C_{T}(2-B)\right)+4\left(1+b_{4}\right)\left(1+C_{T}(2-B)\right)-\left(1-B C_{T}\right)^{2}-4 C_{T}=0 .
$$

The solution to the quartic equation determines the value of $b_{4}$ that is consistent with the pressure gradient which develops in the flow passage between stations one and four due to the thrust, $C_{T}$, applied to the flow.

The angular velocity of the rotor is $\Omega$, and an angular velocity induction factor $a_{2 i}^{\prime}$ is induced in the $i^{\text {th }}$ annular ring at the rotor plane resulting in a whirl velocity of $a_{2 i}^{\prime} r_{i} \Omega$. Generalised BEM analyses for wind turbines have allowed the induced angular velocity to vary in the wake, resulting in an additional factor $a_{4 i}^{\prime}$ in the rotor wake. An additional equation or a similarity relationship e.g. $\frac{a_{2 i}^{\prime}}{a_{2 i}}=\frac{a_{4 i}^{\prime}}{a_{4 i}}[25]$ is required, but in the absence of a physical basis and for simplicity, the generalised approach is not adopted in this work. The incremental torque, $\delta Q_{i}$, on the $i^{\text {th }}$ annular ring can be found in a similar manner to the incremental thrust by considering conversation of angular momentum in an annular ring:

$$
\delta Q_{i}=4 \pi \rho r_{i}^{3} u_{a}\left(1-a_{2 i}\right) \Omega a_{i}^{\prime} \delta r_{i} .
$$

\section{Finite Blockage BEM}

Expressions for the incremental thrust and torque are required to relate blockage-corrected momentum theory to the lift and drag forces acting on the rotor blades. The flow angle between the relative velocity $u_{r e l, i}$ and the direction of rotation of the $i^{\text {th }}$ segment of a blade is defined as $\phi_{i}=\alpha_{i}+\beta_{i}$, where $\alpha_{i}$ is the angle of attack and $\beta_{i}$ is the blade twist angle. Defining the number of rotor blades as $N_{B}$ and the blade element chord length to be $c_{i}$, this gives:

$$
\delta T_{i}=\frac{1}{2} \rho u_{r e l, i}^{2} N_{B} c_{i}\left(C_{l i} \cos \phi_{i}+C_{d i} \sin \phi_{i}\right) \delta r_{i},
$$

where $C_{l i}$ and $C_{d i}$ are the sectional lift and drag coefficients of the blade element respectively. Equating the thrust increment on the $i^{\text {th }}$ annulus required to satisfy momentum conservation, Equation (7), to that generated by the blade increments in the $i^{\text {th }}$ annulus, (17), an expression for $a_{2 i}$ may be found:

$$
a_{2 i}=\frac{\sigma_{i}\left(1-a_{4 i}\right)\left(C_{l i} \cos \phi_{i}+C_{d i} \sin \phi_{i}\right)-\sin ^{2} \phi_{i}\left(b_{4}^{2}+2\left(a_{4 i}+b_{4}\right)-2 a_{4 i}^{2}\right)}{\sigma_{i}\left(1-a_{4 i}\right)\left(C_{l i} \cos \phi_{i}+C_{d i} \sin \phi_{i}\right)}
$$


where $\sigma_{i}=\frac{N_{B} c_{i}}{2 \pi r_{i}}$ is the solidity of the $i^{\text {th }}$ annular ring. Equation (9) is used to update the solution for $a_{4 i}$. The torque may be expressed in terms of the forces on the blade element as:

$$
\delta Q_{i}=\frac{1}{2} \rho u_{r e l, i}^{2} N_{B} c_{i} r_{i}\left(C_{l i} \sin \phi_{i}-C_{d i} \cos \phi_{i}\right) \delta r_{i} .
$$

Eliminating $\delta Q_{i}$ between (16) and (19) yields an expression for the annular angular induction factor:

$$
a_{2 i}^{\prime}=\frac{\sigma_{i}\left(C_{l i} \sin \phi_{i}-C_{d i} \cos \phi_{i}\right)}{4 \sin \phi_{i} \cos \phi_{i}-\sigma_{i}\left(C_{l i} \sin \phi_{i}-C_{d i} \cos \phi_{i}\right)} .
$$

BEM analysis usually assumes that all fluid particles passing through the swept area of a rotor annulus undergo the same loss of momentum, which implies that the rotor has sufficiently many blades to ensure that all fluid particles interact with a blade as they pass through the rotor plane [5]. Corrections exist to account for the effects of a finite number of blades on the rotor performance, such as the Prandtl-type tip loss models [3], which reduces the azimuthal average axial induction factor $a_{2 i}$ to zero as the rotor tip is approached, and has been implemented here (using Glauert's formulation [10]):

$$
F_{i}=\frac{2}{\pi} \arccos e^{-f_{i}}, \quad f_{i}=\frac{N_{B}}{2} \frac{R-r_{i}}{r_{i} \sin \phi_{i}} .
$$

where the tip loss factor $F_{i}=a_{2 i} / a_{b 2 i}$ is the ratio between the azimuthally averaged induction factor, $a_{2 i}$, and the induction factor at the blade position, $a_{b 2 i}$ (the same ratio applies for the other induction factors). The tip loss factor multiplies the azimuthally averaged induction factors $a_{2 i}, a_{4 i}$, and $a_{2 i}^{\prime}$ in the momentum equations, whereas the blade element equations are written in terms of the blade-local induction factors. Consequently, applying the tip loss factor slightly modifies the form of Equations (18) and (20). It should be noted that the Prandtl tip loss model may not describe accurately the flow conditions near the tips of highly blocked tidal turbine rotor blades, as the flow speed in the bypass will be greater than the free stream flow speed. The effect of the volume flux constraint on the interaction between the free stream flow and rotor tips is currently unknown, and further work will be required to assess the suitability of tip loss models for highly blocked turbines.

The blockage-corrected BEM problem can be solved given the specification of the rotor's blockage ratio $B$, tip speed ratio, $\lambda$, and rotor data, such as lift and drag curves with respect to angle of attack, blade twist and solidity data, and the flow speed. The solution procedure of the blockage-corrected BEM model also requires that initial distributions of $a_{2 i}$ and $a_{2 i}^{\prime}$ (blade-local) are specified. Uniform distributions $a_{2 i}=0.3$ and $a_{2 i}^{\prime}=0.01$ are specified herein, which are then used to compute the flow angle:

$$
\tan \phi_{i}=\frac{u_{a}\left(1-a_{2 i}\right)}{r_{i} \Omega\left(1+a_{2 i}^{\prime}\right)} .
$$

The angle of attack is then determined, allowing the lift and drag coefficients to be determined, and consequently the thrust of each blade element to be calculated from Equation (17). The sum of the thrust is then used to determine the rotor thrust coefficient $C_{T}$, which allows the bypass induction factor, $b_{4}$, to be determined from Equation (15). The updated $b_{4}$ is used to calculate the wake induction factor, $a_{4 i}$, from Equation (9). New estimates for $a_{2 i}$ and $a_{2 i}^{\prime}$ are then calculated using Equations (18) and (20) respectively, from which the new flow angle, $\phi_{i}$, is calculated from (22), and the solution iterated. The system of equations is solved iteratively until convergence to a relative error of $10^{-6}$ is achieved.

\section{Comparison to Blade Resolved Simulations}

The confined BEM model derived above is compared to a suite of blade resolved computations performed by Wimshurst and Willden [30, 29]. Wimshurst and Willden analysed the performance in uniform flow of two different tidal turbine rotors, which were designed to maximise turbine power coefficient in blockage ratios of 

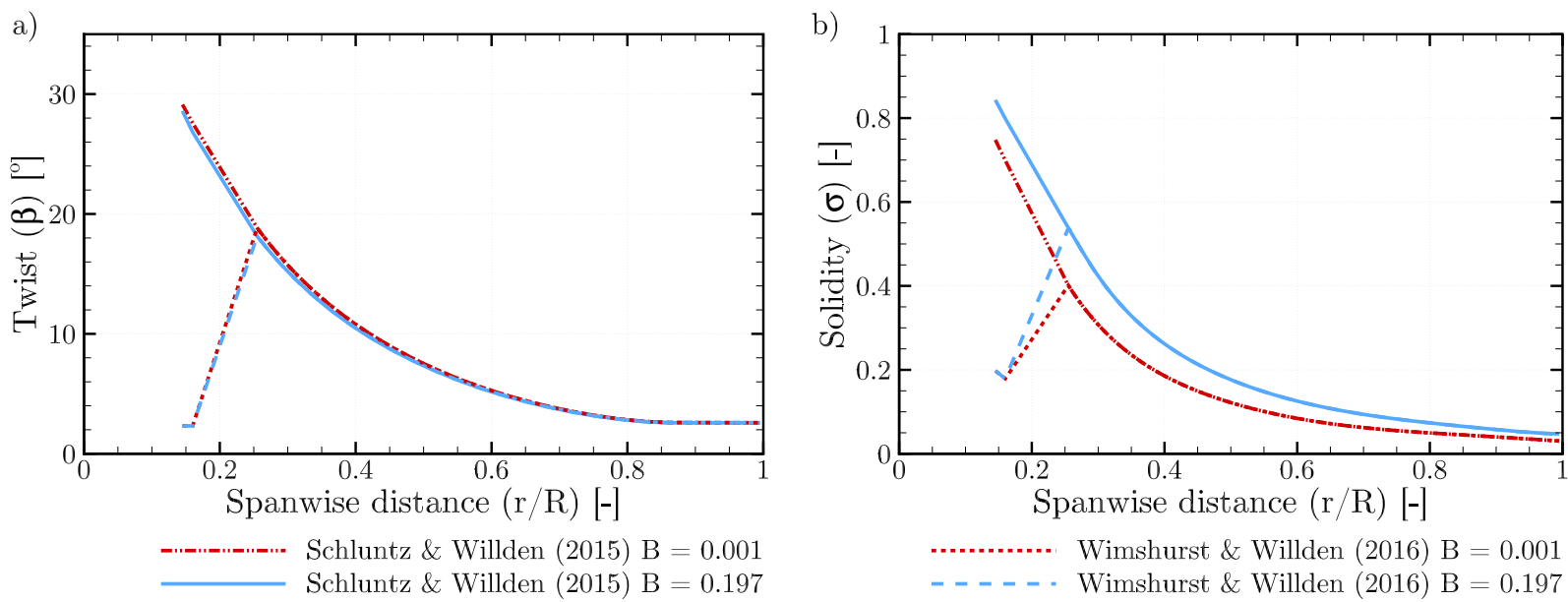

Figure 2: Comparison of the rotor twist (a) and solidity (b) for the blocked and unblocked designs of Schluntz and Willden [21] and modifications to rotor geometry in the blade resolved simulations of Wimshurst and Willden [30]. The rotor radius $R=10 \mathrm{~m}$.

$B=0.010$ (unblocked) and $B=0.196$ (blocked) respectively. Wimshurst and Willden performed the steady Reynolds Averaged Navier Stokes (RANS) blade resolved computations in OpenFOAM, using a multiple reference frame approach to model a $120^{\circ}$ wedge encompassing one blade. The computational domain was divided into two regions to permit a fine resolution near the rotor blade, coupled through an arbitrary mesh interface to a coarser outer domain. The wall-adjacent cells along the blade surface of the inner domain were sized to ensure that the cell centroid lay within the logarithmic law region, so that the dimensionless wall distance lay in the region $30<y^{+}<300$. Turbulence closure was provided using the $k-\omega$ SST turbulence model of Menter [15]. Further details and results of the computations may be found in [30, 29, 32].

Both the blocked and unblocked turbines were designed to operate at a tip speed ratio of $\lambda=5.0$. The rotors, with a diameter $d=20 \mathrm{~m}$, had a nacelle of diameter $d_{h u b}=0.15 d$, and used the Ris $\varnothing$-A1-24 blade section along the entire span of the blade, with the relatively thick aerofoil section, insensitivity to leading edge roughness, and gradual stall transition being considered as good properties for tidal stream turbine applications [30]. The rotors used by Wimshurst and Willden were adapted from earlier designs developed by Schluntz and Willden [20], with modifications made to the blade chord and twist distributions, shown in Figure 2, inboard of the radial position $r / R=0.25$ to blend the aerofoil into a cylinder for attachment to the nacelle. Slight modifications were also made to hold the blade twist constant outboard of $r / R=0.90$. Although the trailing edge was thickened in the blade resolved computations to aid the meshing process, the BEM comparisons presented herein will be computed using 2D aerofoil data at a chord-based Reynolds number $R e=12 \times 10^{6}$ [8] which includes a sharp trailing edge. A study by McNaughton [14] and analysis by Wimshurst and Willden [30] reported that this does not significantly alter the fluid-dynamic performance of the aerofoil.

Comparison is made of thrust and power coefficients predicted for the unblocked rotor design with blade resolved simulations and the analytic confined BEM model in Figure 3 for four different blockage ratios. The confined BEM model substantially under-predicts the integrated rotor quantities at the lowest tip speed ratios simulated $(\lambda \leq 4)$ where significant portions of the blade are expected to be stalled based on the $2 \mathrm{D}$ aerofoil characteristics and calculated angle of attack. The additional spanwise component of flow that develops along a rotating blade (not modelled in BEM theory) has been observed to delay the onset of stall [24], and hence there is a difference between the predictions of the two models. The magnitude of the blade surface pressure gradients that give rise to the spanwise flows depend on the magnitude of the incident flow, which increases with tip speed ratio. It may be possible to improve agreement in this region by correcting the 2D lift and drag polars used in the BEM model by applying a stall delay correction to the data, or extracting the polars at a range of radial locations from the blade resolved computations to account for the effects of 


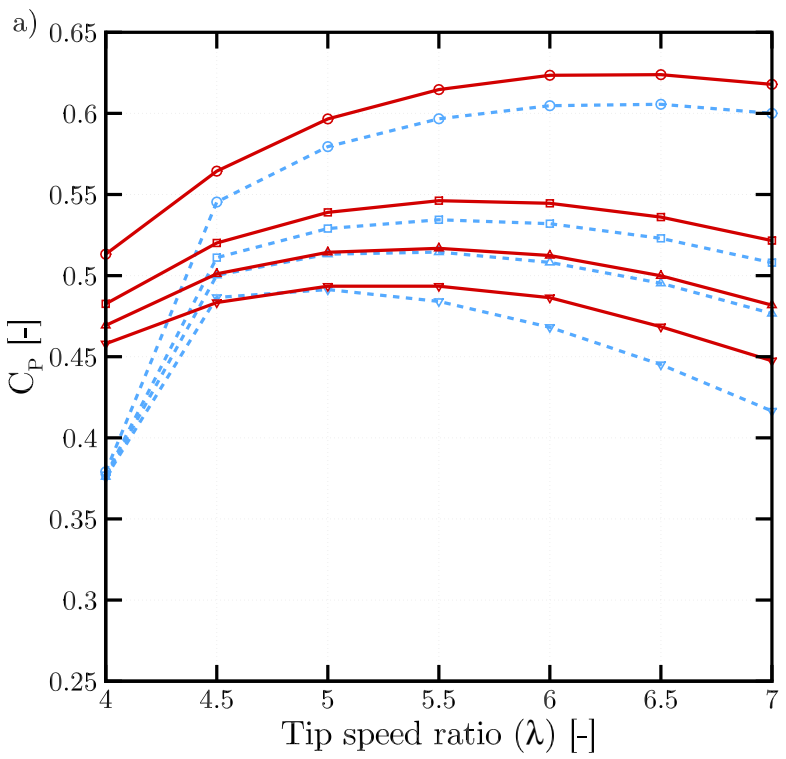

$\longrightarrow$ Blade resolved $\quad \nabla-\mathrm{B}=0.010 \quad \triangle \mathrm{B}=0.065$

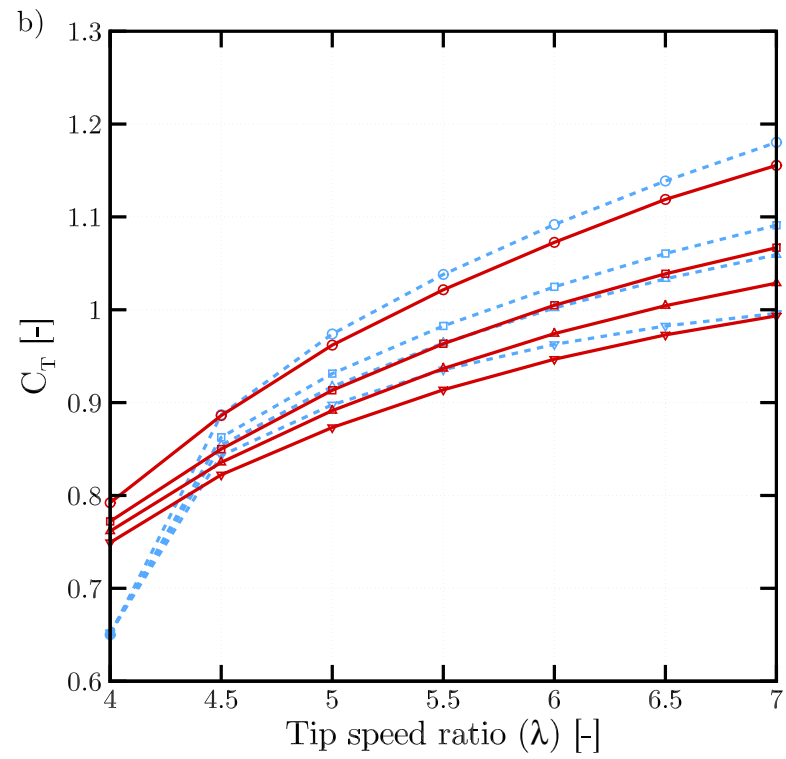

$\square \mathrm{B}=0.098 \quad \square \quad \mathrm{B}=0.196 \quad$ Confined BEM -

Figure 3: Power coefficient, $C_{P}$ (a) and thrust coefficient $C_{T}$ (b) variation with tip speed ratio $\lambda$ for the unblocked rotor in four different blockage ratio domains. The solid red lines indicate results from blade resolved computations, and the dashed blue lines indicate results from the confined BEM model.

spanwise flow.

At higher tip speed ratios, agreement between the BEM model and blade resolved computations is reasonably good for all blockage ratios. Because the angle of attack of the incident flow is small $\left(\alpha<10^{\circ}\right)$, the flow remains attached to the blade surface and the approximation of blade forces using $2 \mathrm{D}$ aerofoil polars (with the Prandtl tip loss model employed) performs reasonably well. The power and thrust coefficients increase with blockage ratio as the increased confinement of the bypass flow around the rotor allows a greater streamwise static pressure drop in the flow passage encompassing the rotor to develop. When operating at a given tip speed ratio $\lambda$, the increased static pressure difference along the flow passage due to increasing blockage ratio results in a higher flow speed through the rotor plane, resulting in larger blade forces and thus increased rotor thrust and power. The relative difference in power coefficient, $C_{P}$, predicted by the confined BEM and blade resolved methods at $\lambda=5.0$ is small, varying from $0.5 \%$ for $B=0.010$ to approximately $3 \%$ when $B=0.196$. For comparison, there is an approximately $20 \%$ change in $C_{P}$ between the two blockage ratios at $\lambda=5.0$. The relative difference in $C_{T}$ reduces from almost $3 \%$ in the lowest blockage case to $1.25 \%$ in the highest blockage case. The relative differences in $C_{P}$ and $C_{T}$ remain within similar magnitudes across the four blockage ratios considered herein as the tip speed ratio varies. The general trend for the BEM model under-predicting the power coefficient and over-predicting the thrust coefficient can be investigated by considering the variation in axial and tangential forces per unit span with radial position.

Figure 4 compares the predictions of axial $\left(F_{a x}\right)$ and tangential $\left(F_{t n}\right)$ force per unit span for the unblocked rotor at a tip speed ratio $\lambda=5.0$ in a domain of blockage $B=0.01$. Close agreement is observed between the confined BEM and blade resolved predictions for $F_{a x}$, with discrepancies near the root section (inboard of $r / R=0.25)$ and near the blade tip $(r / R>0.9)$. The differences inboard of $r / R=0.25$ arise as a result of slight differences in the rotor geometry used in the two models, as the BEM model did not implement all the geometrical modifications employed in the blade resolved computations in order to taper the blades to a cylindrical attachment to the nacelle. The relative difference in axial force per unit span between the confined BEM and blade resolved models is small for the majority of the blade span beyond $r / R>0.25$, although the agreement between the two models diverges near the blade tip, with the confined BEM model 

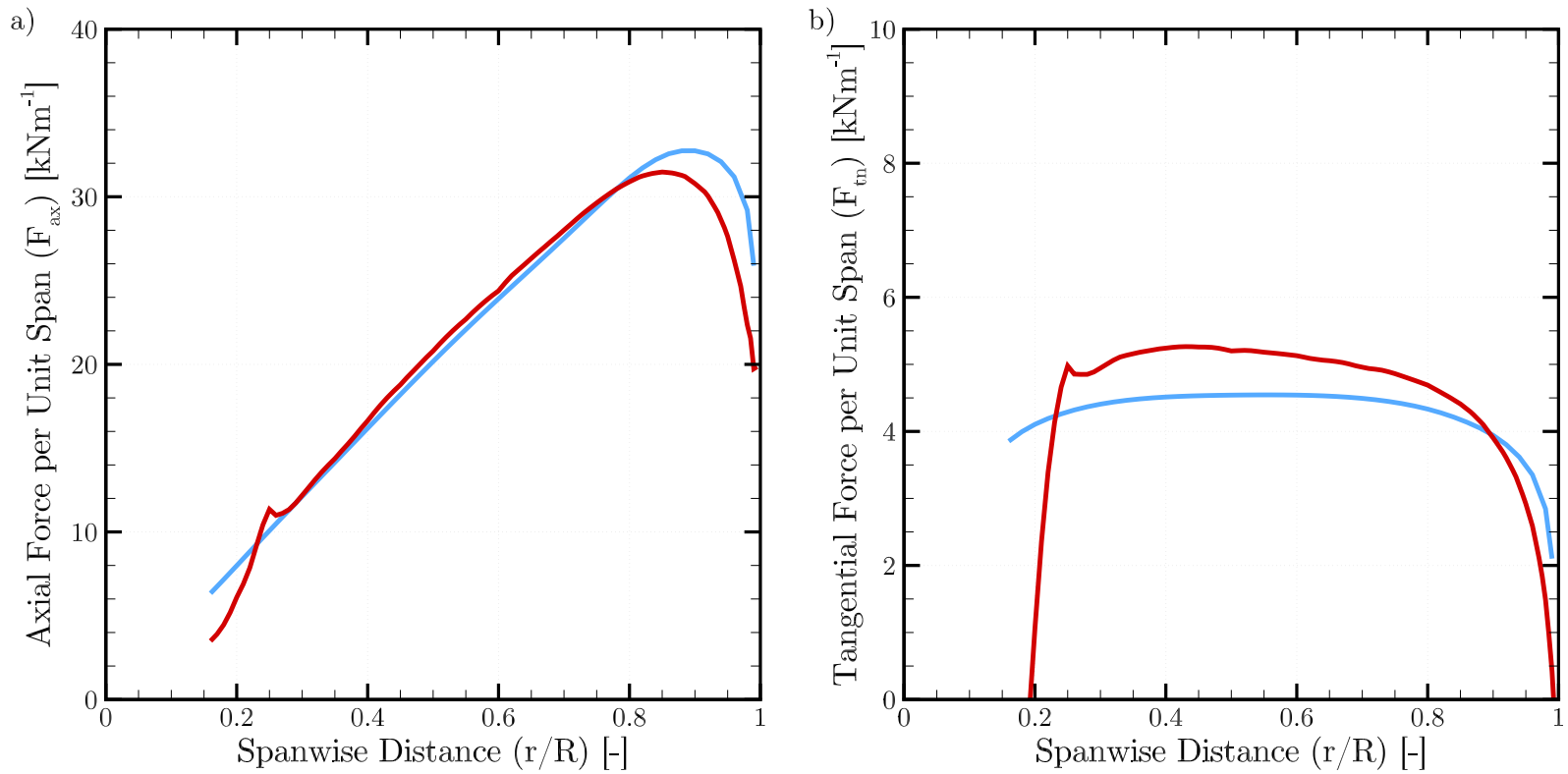

Figure 4: Spanwise variation in axial force $F_{a x}$ (a) and tangential force $F_{t n}$ (b) per unit span for the unblocked rotor operating at $\lambda=5.00$ in $B=0.01$. Results from the blade resolved computations of [30] are shown in red, and the blue lines indicate results from the confined BEM method.

over-predicting $F_{a x}$. The reduction in blade forces near the tip in the BEM model is driven by the Prandtl tip loss model, and comparison between $F_{a x}$ in the confined BEM and blade resolved results suggests that improved agreement in integrated thrust could be achieved if a tip loss model that more closely accounts for the greater spanwise flow induced load reduction at the blade tips were applied, such as that of Shen and Sørensen [22] or Wimshurst and Willden [31]. The tip loss effects also depend on flow confinement, as shown in the discrepancy between integrated thrust and power coefficients between the blade resolved and BEM solutions in Figure 4. Whereas the Prandtl tip loss model returns the azimuthally averaged axial flow speed at the blade tip to the freestream flow speed, one effect of flow confinement is that the bypass flow around the rotor is accelerated to greater than the freestream speed. This discrepancy increases with blockage ratio, although the effect on the rotor thrust and power is differs due to the difference in how the resultant force vector rotates near the tip, as discussed in [32]. This could be accounted for through improved tip loss modelling for confined flow environments where the bypass flow is accelerated.

As shown in Figure 4, the confined BEM model generally predicts a lower tangential force per unit span than that simulated in the blade resolved computations, which gives rise to the under-prediction of integrated rotor power as shown previously in Figure 3. The largest relative differences in $F_{t n}$ between the confined BEM and blade resolved models are observed at smaller values of $r / R$, with a maximum difference of c. $14 \%$ occurring at $r / R=0.4$. This phenomenon has also been observed for wind turbines (e.g. [23]), and arises because the effects of three dimensional flow along the blades such as rotational augmentation are not represented in the $2 \mathrm{D}$ aerofoil data. As the magnitude of $F_{t n}$ is smaller than $F_{a x}$, the impact of these three dimensional flow effects is proportionally larger in the tangential direction. Inboard, the contribution to rotor torque is smaller than regions further outboard, where agreement between the two models is better, and hence there is better overall agreement for rotor torque (and hence power) than with $F_{t n}$ between the two models. Although both modelling approaches predict a reducing $F_{t n}$ near the tip region, the BEM model predicts a higher value than the blade resolved computations for $r / R>0.9$, which is a consequence of an inadequate rate of reduction of blade forces with span through the Prandtl tip loss model. A more sophisticated tip loss model, which would reduce $F_{t n}$ for $r / R>0.9$, could result in a reduction in $C_{P}$ which in turn may reduce agreement between the BEM and blade resolved results. 


\section{Rotor Performance in Power Capping}

Power capping will be an important feature of tidal turbine design and operation in order to balance the capital cost of the turbine and generator with the power (and therefore revenue) available from the fluctuating tide. Between the cut-in flow speed (here assumed $0.5 \mathrm{~ms}^{-1}$ ) and rated flow speed, the turbine operates at the peak power coefficient and corresponding tip speed ratio in order to maximise power. The unblocked rotor design, with diameter $d=20 \mathrm{~m}$, used by Wimshurst and Willden [30] is adopted, and modelled in water of depth $h=2 d$. It is assumed the rotor is deployed in a side-by-side fence configuration with other rotors, with an inter-turbine spacing ratio $s / d=1$, giving a blockage ratio $B=0.196$. Figure 5 shows that a peak power coefficient of $C_{P}=0.61$ is predicted with the confined BEM model, compared to $C_{P}=0.49$ with unconfined BEM. The confined flow passage surrounding the turbine can support the development of a larger static pressure difference across the rotor, and thus greater rotor thrust. This effect is modelled in the confined BEM method, and consequently the rotor operates at higher $\lambda=6.5$ in order to maximise $C_{P}$, than when analysed with the unconfined BEM model, where $\lambda=5.0$.

Setting the turbine rated power to be $1 \mathrm{MW}$, the rated flow speed is determined to be $u_{r}=2.17 \mathrm{~ms}^{-1}$, as compared to $u_{r}=2.33 \mathrm{~ms}^{-1}$, when the effects of flow confinement are accounted for. The difference in rated flow speeds in the two cases has important practical consequences, as it means that turbines operating in a blocked environment achieve rated flow speed at a lower speed, and experience higher thrusts, than predicted from an unblocked analysis. Above the rated flow speed, the power coefficient must reduce as $\left(u_{r} / u_{a}\right)^{3}$ in order to maintain rated power until the cut-out flow speed (here taken as $3.5 \mathrm{~ms}^{-1}$ ) at which $C_{P}=0.15$. Common power capping strategies for wind turbines can be broadly grouped into two categories: fixed pitch and variable pitch [5]. In the fixed pitch case, rotor blades are installed at a prescribed pitch angle, and the turbine is controlled by altering its rotational speed. In the variable pitch case, the pitch of the rotor blades can vary, providing an additional degree of freedom to control the turbine. Two dimensional aerofoil theory, used in the BEM model, does not provide a good approximation to flow around the turbine blades in stall, so the analysis herein is conducted assuming that the turbine is operated in overspeed conditions (where the flow remains attached) in the fixed pitch case, and pitch to feather in the variable pitch case.

The analytic BEM model is used to explore the effect of the different power capping control strategies on rotor performance. The required variation in power coefficient with flow speed is independent of control strategy and is shown in Figure 5. The turbine operates at the peak power coefficient until the rated flow speed is achieved, which must then reduce as the flow speed increases further. The reduction in power coefficient is achieved by varying the rotational speed of the fixed pitch rotor, increasing tip speed ratio in order to avoid stall. This is discussed further below. For simplicity, it is assumed that the variable pitch rotor operates at a constant rotational speed once rated power is achieved, which corresponds to the optimal rotational speed of the generator, and thus $\lambda \propto\left(u_{r} / u_{a}\right)$ for $u_{a}>u_{r}$. Other power control strategies, such as variable pitch and variable speed, and fixed pitch and fixed speed, are also possible, but are beyond the scope of the present work. Hence, we adopt the shorthand 'fixed pitch' to refer only to the fixed pitch, variable speed case, and 'variable pitch' to refer only to the variable pitch, fixed speed case.

\subsection{Fixed Pitch, Variable Speed}

Control of fixed pitch turbines during rated power operation is achieved by varying the rotational speed of the rotor to move away from the peak power coefficient. As seen in Figure 6, the peak power coefficient reduces as the tip speed ratio moves away from the optimal value for the blocked $(\lambda=6.5)$ and unblocked $(\lambda=5.0)$ cases. The flow over the blades may stall at low tip speed ratio and is difficult to represent accurately in BEM theory based on two dimensional aerofoil data. Consequently, only overspeed operation is analysed herein for a fixed pitch turbine, although it should be noted that the minimum suction surface pressure on the rotor blades reduces as $\lambda$ increases, making cavitation more likely under overspeed control.

The required change in tip speed ratio for the fixed pitch rotor as flow speed increases is shown in Figure 5. A greater change in tip speed ratio is required in confined flow case, as rotor starts with a higher value of $C_{P}$ at a lower rated flow speed, and hence a greater change in operating condition is required than in 

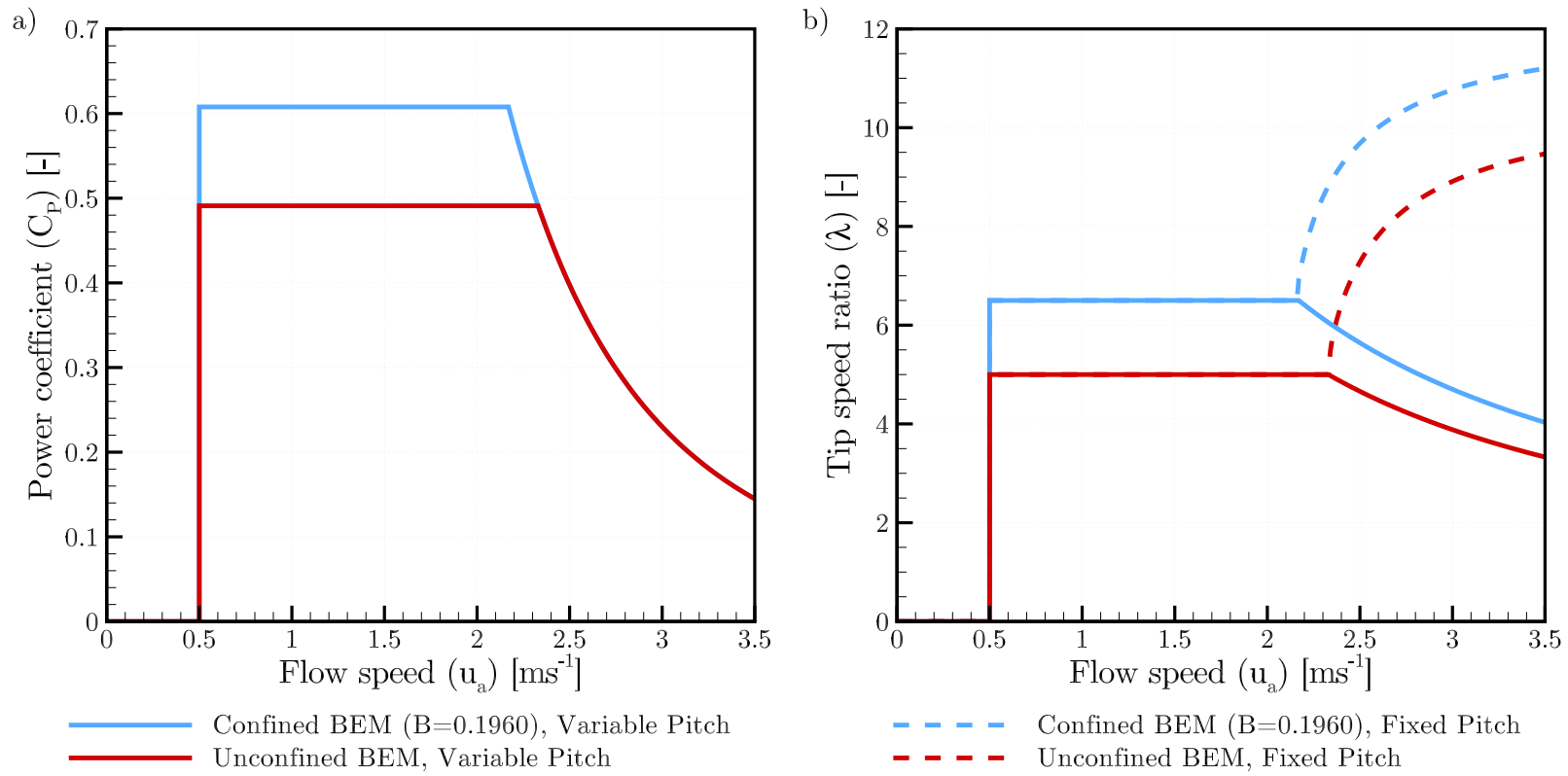

Figure 5: (a) Required variation in power coefficient, $C_{P}$, and (b) tip speed ratio, $\lambda$, with flow speed $u_{a}$ for a variable pitch (solid lines) and a fixed pitch (dashed lines) turbine operating in a blockage ratio $B=0.1960$ using confined (blue) and unconfined (red) analytic BEM theory. Note in (a) that the variable pitch and fixed pitch curves are the same.

unconfined flow. The corresponding change in thrust coefficient, $C_{T}$, can be determined from the confined and unconfined analytic models and is shown in Figure $6 . C_{T}$ is constant and corresponds to that required to maximise $C_{P}$ between cut-in and rated flow speed, after which there is an initially rapid increase in $C_{T}$ as the rated flow speed is exceeded and $\lambda$ increases, slowing as the flow speed increases further. $C_{T}$ is under-predicted between cut-in and rated flow speed in the unconfined case, as compared to when flow confinement is considered $\left(C_{T}=0.90\right.$ vs. $C_{T}=1.14$ respectively), as the increase in thrust that occurs due to the streamwise static pressure difference that develops in the flow passage is not modelled.

This combination of lower rated flow speed and large required change in $\lambda$ in the confined flow case, means that $C_{T}$ increases more significantly, both relatively and in absolute magnitude, than in unconfined flow to a final value of $C_{T}=1.54$ vs. $C_{T}=1.01$ respectively. Consequently, the unconfined analysis of the rotor significantly underestimates the peak loads by approximately $50 \%$ at cut-out (3.02MN compared to $1.97 \mathrm{MN}$ ). Although the effect of blockage results in an increased turbine power coefficient, and thus a given rated power can be achieved at lower flow speeds, there is also an increase in thrust loading on the turbine, which can be particularly significant for fixed pitch turbines. Thus, not accounting for the effects of blockage on turbine loading may have a significant impact on the structural integrity and fatigue life of fixed-pitch tidal turbines.

\subsection{Variable Pitch, Fixed Speed}

During rated power operation, variable pitch turbines alter the pitch of the turbine blades to change the angle of attack and thereby reduce rotor power. For simplicity, it is assumed here that above rated flow speed the rotational speed of the rotor is fixed at that achieved at rated power, although in the general case this too, in principle, may be changed in order to optimise rotor performance. Although variable pitch-fixed rotational speed operation defines a single target $C_{P}-\lambda$ trajectory for power capping, this may itself be achieved by either increasing the pitch of the blades (to feather) or decreasing the blade pitch (to stall), to move through a family of $C_{P}-\lambda$ curves such as those shown in Figure 7. Pitch-to-feather only is investigated herein, as stall conditions are not accurately modelled with 2D aerofoils in BEM theory.

Increasing the blade pitch angle results in a $C_{P}-\lambda$ curve in which peak $C_{P}$ is achieved at lower values of $\lambda$ and envelope width is reduced. The $C_{P}-\lambda$ trajectory required during power capping, shown in red, is 

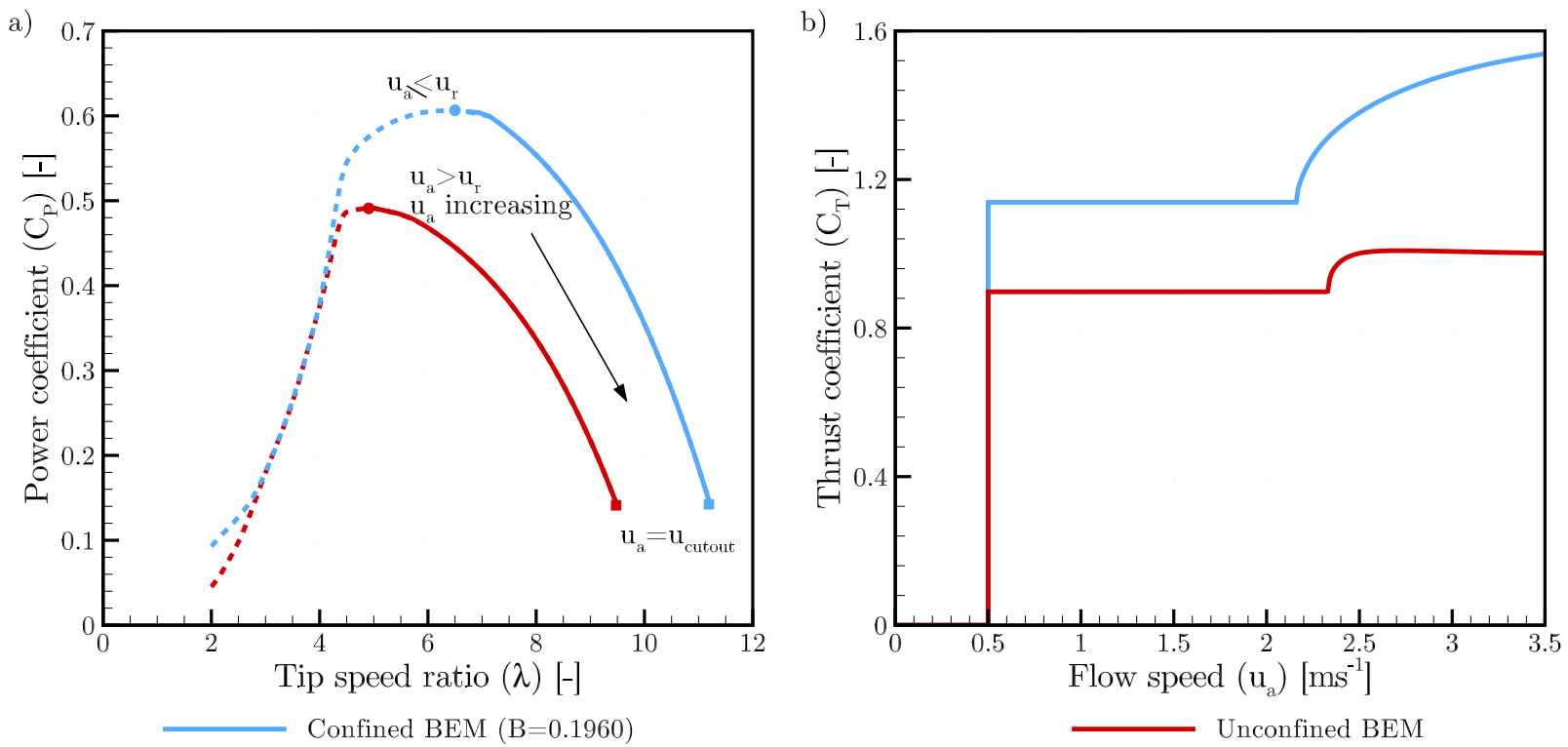

Figure 6: (a) Power coefficient variation with tip speed ratio and (b) thrust coefficient variation with flow speed for the fixed pitch turbine in confined (blue) and unconfined (red) flow, with inter-turbine spacing ratio $s / d=1(B=0.1960)$. The peak power coefficient is denoted with a circle, and the required change in $C_{P}$ as flow speed increases above rated power is shown with a solid line.

similar, but not the same for the blocked and unblocked cases. The blocked case starts from a higher initial $C_{P}$ and $\lambda$, and although the value of $C_{P}$ at the cut-out flow speed is the same for the two cases, the rotor in the blocked domain operates at higher $\lambda$ as the rotational speed (determined by $\lambda$ required for maximum $\left.C_{P}\right)$ is higher $\left(\Omega=1.41 \mathrm{rad} \mathrm{s}^{-1}\right.$ vs. $\Omega=1.17 \mathrm{rad} \mathrm{s}^{-1}$ ). As shown in Figure 7 , a smaller change in blade pitch angle is required for the rotor in the confined domain $\left(16^{\circ}\right)$ as compared to the unconfined domain $\left(18^{\circ}\right)$ between the rated and cut-out flow speeds. The variation in $C_{T}$ during power capping is determined by evaluating $C_{P}-\lambda$ curves for a range of blade pitch angles and matching the curves to the required $C_{P}-\lambda$ trajectory, resulting in a pitch control strategy and ensuing thrust variation; see Figure 8.

The thrust coefficient between cut-in and rated flow speed is higher in confined flows $\left(C_{T}=1.14\right.$ vs. $C_{T}=$ 0.90). As the rated flow speed is lower in confined flows, $u_{r}=2.17 \mathrm{~ms}^{-1}, C_{T}$ starts reducing at lower flow speeds when flow confinement effects are considered. The thrust coefficient predicted in the confined flow model reduces below that predicted with the unconfined BEM model at a flow speed $u_{a}=2.28 \mathrm{~ms}^{-1}$. The thrust coefficient in the unconfined analytic model begins to reduce once the rated speed $u_{r}=2.33 \mathrm{~ms}^{-1}$ is achieved, with the difference between the confined and unconfined BEM models reducing as flow speed increases. There is a difference of $C_{T}=0.01$ at the cut-out flow speed $u_{a}=3.50 \mathrm{~ms}^{-1}$, with the thrust of the rotor in the confined domain being slightly lower than that in the unconfined domain. The differences arise as a result of the higher rotational speed of the rotor in the blocked domain, which is determined as a function of the operating conditions at the peak power point. The effect of blockage on the rotor thrust reduces above rated flow speed, as when the rotor thrust is low there is little acceleration of the bypass flow around the turbine and consequently little change in the static pressure difference that develops in the flow passage. Consequently, there is only a small difference in the blade pitch angle at cut-out for the confined and unconfined cases, $16^{\circ}$ and $18^{\circ}$ respectively.

\subsection{Turbine Parameterisation Within Farm-scale Models}

The combined $C_{P}$ and $C_{T}$ vs. flow speed predictions provide the necessary coupling between turbine performance characteristics and the flow conditions to parameterise simplified models of turbines to be 

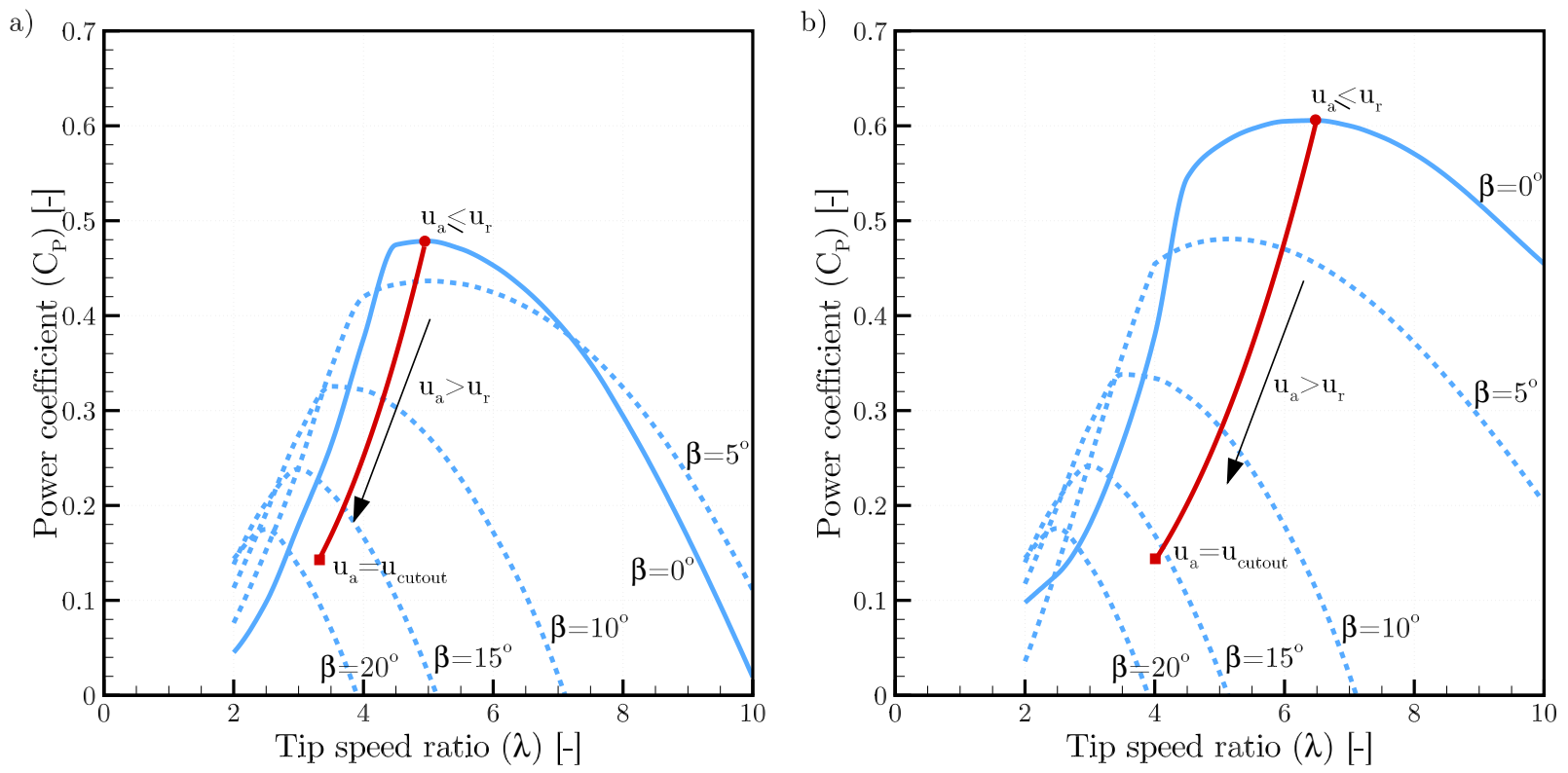

Figure 7: Power coefficient $C_{P}$ vs. tip speed ratio $\lambda$ predicted for unconfined BEM (a) and confined BEM (b) with four positive pitch to feather angles $\beta$ (dashed blue lines), and target $C_{P}-\lambda$ curve for the variable pitch (solid red line) power capping regime. Inter-turbine spacing ratio is $s / d=1(B=0.1960)$, and prior to power capping, the turbine operates at the peak power coefficient, indicated with a circle.

implemented within analytic and computational models of tidal turbine arrays or farm planning models. In particular, this coupling may be used to overcome the difficulties of parameterising tidal turbines in depthaveraged simulations, as discussed in [12]. Determining the turbine thrust is also important for predicting the impact that energy extraction will have on the tidal resource. The greater levels of thrust required by turbines in the fixed pitch case indicates that more momentum will be removed from the flow, and therefore a greater impact on the surrounding environment is expected than for pitch to feather turbines which apply c. $16 \%$ of the thrust to yield the same power at the highest flow speeds. Thus, neglecting the effects of flow confinement will lead to mis-estimation of turbine thrust and consequently the impact of energy extraction on the tidal resource. Additionally, this will have important implications for the structural design of turbines, discussed further below.

\subsection{Bending Moment}

An important design consideration is the compromise between hydrodynamic efficiency, for which thinner blades are preferred, and structural integrity, which requires thicker blade sections, particularly near the blade root. Following Burton et al. [5], it is possible to determine the forces acting on the blade in the in-plane and out-of-plane directions. These forces, acting at a distance from the blade root, generate bending moments that must be resisted by the structural design of the blade. The out-of-plane force acts in the direction of the thrust force, and can be expressed by rearranging Equations (8) and (17) for the force per unit length on an individual blade, $F_{x i}$ at the $i^{\text {th }}$ radial position:

$$
F_{x i}=\frac{1}{2} \rho u_{r e l, i}^{2}\left(C_{l i} \cos \phi_{i}+C_{d i} \sin \phi_{i}\right) c_{i}=\frac{\rho \pi u_{a}^{2}}{N_{B}}\left(b_{4}^{2}+2 b_{4}+2 a_{4 i} F_{i}\left(1-a_{4 i} F_{i}\right)\right)\left(\frac{r_{i}}{R}\right) R,
$$

where $F_{i}$ is the Prandtl tip loss factor which is applied to account for the reduction in induction factor towards the tips. Similarly, the force per unit length acting on a blade in the in-plane direction, $F_{y i}$, (defined positive in the torque opposing direction) can be found with Equations (16) and (19):

$$
-F_{y i}=\frac{1}{2} \rho u_{r e l, i}^{2}\left(C_{l i} \sin \phi_{i}-C_{d i} \cos \phi_{i}\right) c_{i}=4 \pi \rho \lambda u_{a}^{2}\left(1-a_{2 i} F_{i}\right) a_{i}^{\prime} \frac{F_{i}}{N_{B}}\left(\frac{r_{i}}{R}\right)^{2} R .
$$




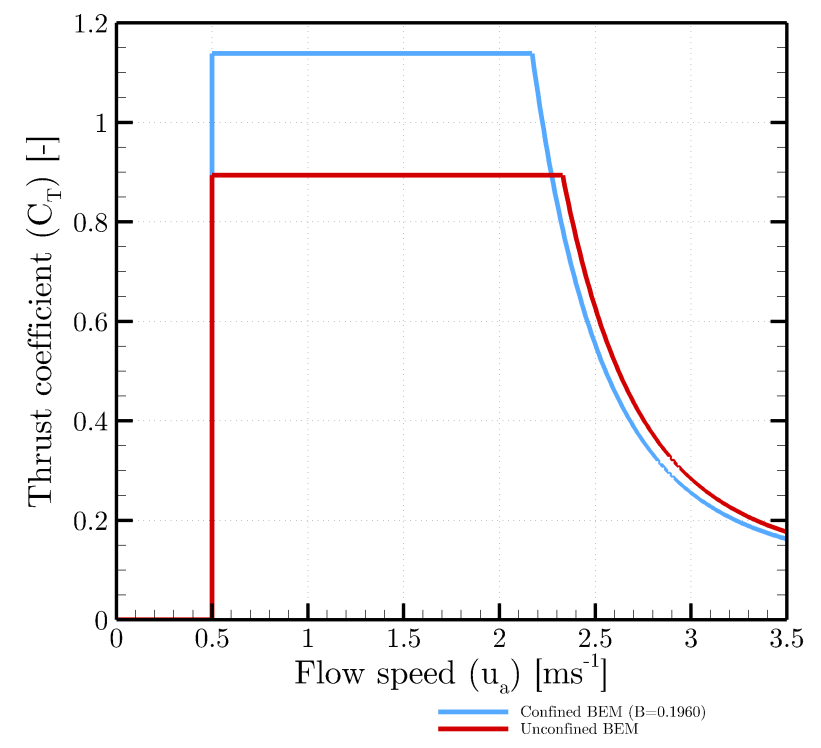

Figure 8: Thrust coefficient $C_{T}$ vs. flow speed $u_{a}$ for the pitch to feather turbine as predicted by the confined (blue) and unconfined (red) analytic BEM models. Inter-turbine spacing is $s / d=1(B=0.1960)$, and prior to the rated flow speed the turbines operate at the peak power coefficient and corresponding thrust coefficient.

The quantities in Equations (23) and (24) are computed in the course of solving the BEM model, and therefore do not represent significant additional computational costs. Blade root edgewise, $M_{e d g e}$, and flapwise, $M_{\text {flap }}$, bending moments are calculated, following Moriarty [16], by integrating the in-plane and out-of-plane bending moments along the blade span and then transforming the moments into the blade root edgewise and flapwise coordinate system:

$$
M_{\text {edge }}=\int_{r_{\text {hub }}}^{R} F_{x} r d r \sin \beta_{r}+\int_{r_{h u b}}^{R} F_{y} r d r \cos \beta_{r}, \quad M_{\text {flap }}=\int_{r_{\text {hub }}}^{R} F_{x} r d r \cos \beta_{r}-\int_{r_{\text {hub }}}^{R} F_{y} r d r \sin \beta_{r}
$$

where $\beta_{r}$ is the blade root pitch angle.

Root bending moment increases at a rate proportional to $u_{a}^{2}$ below rated flow speed, as shown in Figure 9 , with the flapwise root bending moment being larger than the edgewise root bending moment. Root bending moments also increase with blockage due to the increased rotor loads that arise from the larger streamwise static pressure difference in the channel. Above rated flow speed, edgewise and flapwise root bending moments increase in the fixed pitch case, and decrease in the variable pitch case.

Flapwise and edgewise root bending moments increase significantly in the fixed pitch case above rated flow speed, as shown in Figure 9. Overspeed control of the turbines above the rated flow speed results in a reduction of angle of attack, $\alpha$, on the blades, and hence the lift to drag ratio of the aerofoil significantly decreases. Consequently, whilst rotor torque reduces in order to maintain rated power, there is a significant increase in rotor thrust, which dominates the contributions to the in-plane and out-of-plane blade forces. The rate of increase in flapwise and edgewise root bending moment reduces at higher flow speeds as the required rate of change of tip speed ratio with flow speed reduces. Above rated flow speed, which occurs at a lower flow speed when blockage constrains flow expansion, the difference in turbine control has an important effect on the change in root bending moment, with a larger change in tip speed ratio, and thus change in angle of attack required as blockage increases.

Pitching the blades to feather above rated flow speed results in a reduction in flapwise and edgewise root bending moment as a result of the reduction in tip speed ratio and rotor thrust during power capping, resulting in smaller in-plane and out-of-plane forces acting on the rotor blades. Figure 9 shows that the peak 

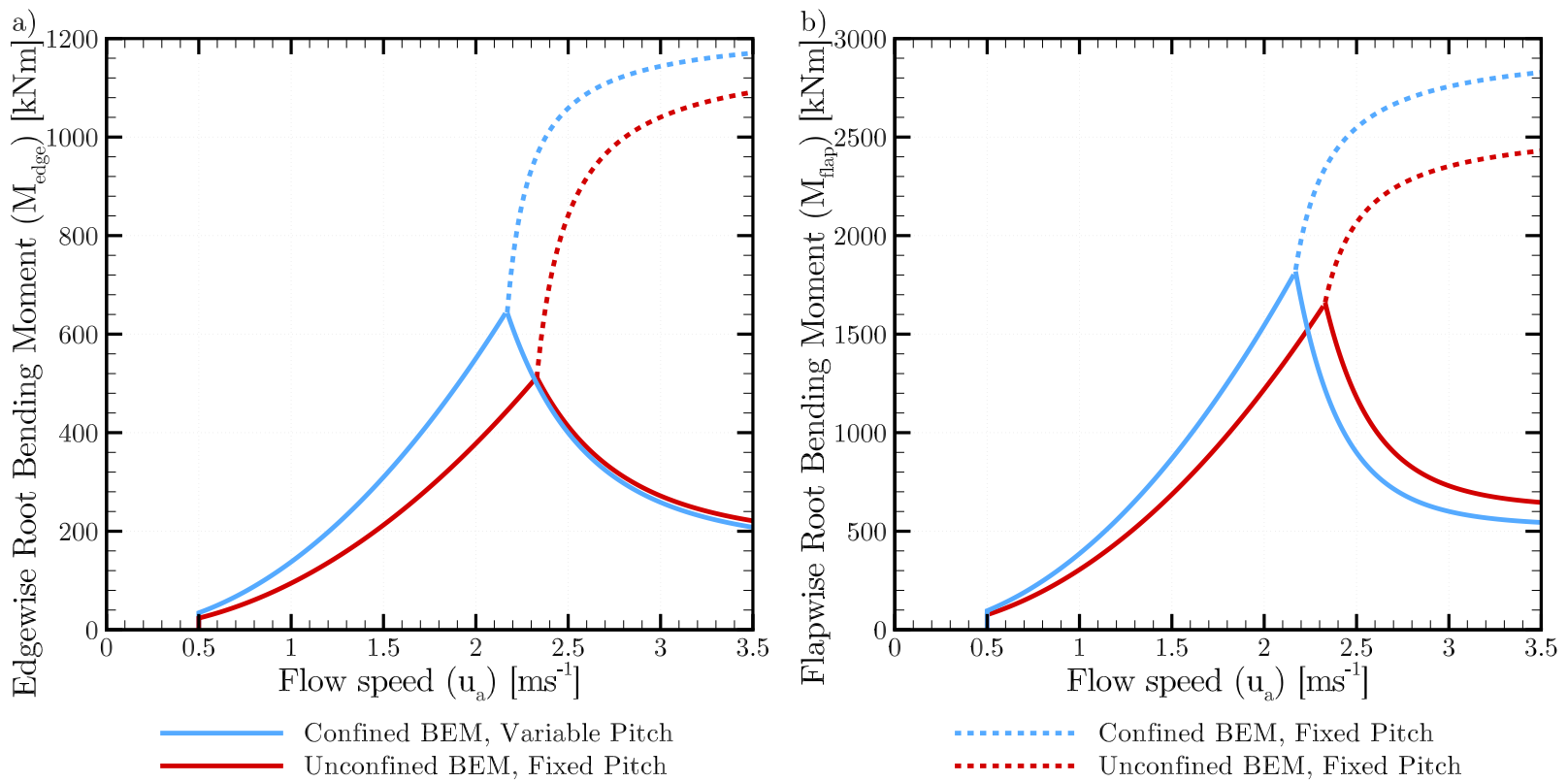

Figure 9: (a) Edgewise $\left(M_{\text {edge }}\right)$ and (b) flapwise $\left(M_{\text {flap }}\right)$ root bending moment vs. flow speed $u_{a}$ for fixed (dashed) and variable (solid) pitch operation, as predicted by confined (blue) and unconfined (red) analytic BEM. Inter-turbine spacing is $s / d=1(B=0.1960)$, and prior to rated flow speed the turbines operate at the peak power coefficient and corresponding thrust coefficient.

flapwise and edgewise root bending moments are larger and occur at lower flow speed in confined rather than unconfined flows due to the larger blade forces that occur when the turbine operates in blocked conditions. The difference in peak flapwise root bending moment between the two cases $(1784 \mathrm{kNm}$ vs. $1641 \mathrm{kNm}$ respectively) is relatively smaller than the difference in peak edgewise root bending moment ( $641 \mathrm{kNm}$ vs. $505 \mathrm{kNm}$ ) due to the significantly larger blade forces that arise in blocked conditions when the rotor operates at peak power. Although the peak edgewise root bending moment is larger and occurs at a lower flow speed, in blocked flows (Figure 9 a), the confined and unconfined models predict a similar magnitude for $M_{\text {edge }}$ at higher flow speeds, with the reduction in tangential (torque) direction forces dominating the change in edgewise bending moment (see Equation 25). The reduction in flapwise root bending moment is dominated by the change in axial (thrust) forces above the rated flow speed, with the difference in rotational speed of the rotor in the two models, determined by conditions at the rated flow speed, contributing to the difference in $M_{\text {flap }}$ between the models.

The differences in flapwise and edgewise root bending moment above rated flow speed between the fixed pitch and variable pitch power capping cases results in maximum loading conditions that must be considered in the structural analysis of a turbine. The maximum loading condition in the fixed pitch case occurs at the highest flow speeds due to the increase in blade loads and root bending moment when the turbine operates in overspeed control. Conversely, the reduction in blade loading and root bending moment above rated flow speed for the turbine with pitch-to-feather power capping implemented means that the maximum loading condition occurs at the rated flow speed for the turbine. In both power capping control cases, operating in a confined flow environment results in higher blade loading and root bending moments at lower flow speeds than are predicted in an unconfined flow. Consequently, higher blade loads and moments are encountered at lower speeds in confined flows, which increases how quickly fatigue damage is accumulated by the rotor blades. 


\section{Conclusions}

Blade element momentum theory has been extended to account analytically for the effects of confined flow passages on the momentum equations. Momentum removal from an unbounded flow field, as is typically assumed for wind turbines, results only in a velocity deficit in the wake of the turbine. However, in a volumeconstrained flow field a streamwise static pressure difference develops as a result of momentum removal from the flow, allowing turbines to exert a greater thrust and thereby increasing the peak power coefficient that can be achieved as the blockage ratio increases. The unconfined BEM equations have been modified by accounting for the effects of blockage in the momentum equations of the theory. The velocity deficit in the far wake, relative to that at the turbine plane, is a function of the blockage ratio, and the equations are closed by relating the far wake core velocity, far wake bypass velocity, the static pressure and the turbine thrust using the linear momentum actuator disc model proposed by Garrett and Cummins [9].

The analytic confined BEM model was compared to blade boundary layer resolved computations of Wimshurst and Willden [30] predicting the rotor thrust and power coefficients across a range of inter-turbine spacing (blockage) ratios, as well as through comparison of spanwise variation in axial and tangential blade forces. As the blockage ratio was increased, both the blade resolved computations and the confined BEM model predicted increases in the power and thrust coefficients, with the confined BEM model overestimating rotor thrust by approximately $3 \%$, and underestimating power by a similar magnitude at design conditions. By comparison, an unconfined (wind turbine) BEM analysis does not predict the increase in thrust and power coefficient as the blockage ratio varies. Good agreement was achieved for the spanwise variation in axial force per unit span between the confined BEM and blade resolved models, with differences at inboard locations $(r / R<0.25)$ arising from the modification of rotor geometry in the blade resolved simulations, and differences near the tips $(r / R>0.9)$ being affected by the tip loss model that was employed. There were larger discrepancies in the prediction of tangential force per unit span between the blade-resolved and BEM models because the relative importance of $3 \mathrm{D}$ flow phenomenon not represented in the 2D aerofoil characteristics employed in BEM models, such as spanwise flow along the blades, is greater.

The confined BEM model provides a tool for the rapid assessment of turbines in a range of different operating conditions. Two different control strategies were investigated; fixed pitch power capping with variable rotational speed, and variable pitch power capping with fixed rotational speed. The effects of the power capping strategies on the thrust applied to the flow, affecting the tidal resource, and the edgewise and flapwise root bending moments, affecting the structural loads on the turbines, were investigated and compared to the predictions of an unconfined (wind turbine) BEM model.

Comparisons between the confined BEM model and an unconfined (unblocked) BEM model show that blockage causes the device loads and power to increase for realistic blockage ratios. Consequently, the root bending moments, and therefore structural requirements of the rotor blades, are higher in confined flows below rated flow speed. Blockage also affects how the bending moments change with flow speed once rated power is achieved. Specifically, rated power is reached at a lower flow speed and more often through the spring-neap tidal cycle, and the peak loads are higher in blocked flows. As a result of the higher blade loads, the fatigue damage rates change for both fixed and variable pitch devices as the effect of blockage increases.

A fixed pitch power capping strategy offers mechanical simplicity, which has attendant reductions in cost, but was found to result in an increase in rotor thrust and edgewise and flapwise root bending moments when overspeed control is used, presenting structural challenges. Reducing the rotational speed at fixed pitch, leading to stall along the blade, could be used as an alternative control strategy. The rotor thrust coefficient reduces at lower rotational speeds, although improved two-dimensional aerofoil data, including stall delay, would be required to fully analyse this operational regime. A variable pitch power capping strategy is more mechanically complex, but results in a decrease in rotor thrust and root bending moments, which may be structurally beneficial. Furthermore, the reduction in thrust is important with respect to minimising the overall impacts on the tidal environment, and the smaller structural loads may improve device lifespan. 


\section{Acknowledgements}

The authors wish to acknowledge the support of the Oxford Martin School. The authors are grateful to A. Wimshurst for providing the data from the blade resolved simulations for comparison in this work.

\section{Appendix A. Streamtube expansion ratio}

Single turbine Reynolds-Averaged Navier Stokes (RANS) embedded Blade Element Actuator Disk (BEAD) simulations, as described in Vogel and Willden [26], were performed to investigate the variation of the expansion ratio $\left(1-a_{2 i}\right) /\left(1-a_{4 i}\right)$ with blockage and turbine thrust. The numerical simulations were performed as steady computations in ANSYS Fluent 15.0, which uses a finite volume method to solve the 3D incompressible RANS equations. The $k-\omega$ SST model was used to provide turbulence closure.

The turbine was modelled using the 'porous jump' boundary condition in Fluent which allows a streamwise static pressure discontinuity and swirl velocity to be imposed on the flow. Blade element theory was used to relate the simulated flow field to the turbine forces. The Prandtl tip loss model was applied to account for the tip loss effects near the edge of the simulated actuator disk. The turbine was simulated in a cylindrical domain that extended $10 d$ upstream and $20 d$ downstream of the rotor plane in the $x$ direction. The domain radius was adjusted to achieve three different blockage ratios: $B=0.0001, B=0.0507, B=0.1960$. Slip wall boundary conditions were applied to the lateral boundaries. The working fluid was seawater $\left(\rho=1025 \mathrm{kgm}^{-3}\right)$, with a dynamic viscosity $\mu=0.001$ Pa.s. A uniform velocity profile of $u_{a}=2 \mathrm{~ms}^{-1}$ was applied to the inlet, and the outlet prescribed to maintain constant gauge pressure. The turbulence intensity in the domain decreased from c. $15 \%$ at the inlet boundary to c. $4 \%$ at the rotor plane, as there was no shear in the domain. A block-structured hexahedral meshing strategy was used to discretise the computational domain. Details of the mesh convergence study may be found in Vogel and Willden [26], where the final resolution of the single turbine mesh contained approximately $2.5 \mathrm{~m}$ cells and had a minimum cell dimension of $d / 200$ at the rotor plane.

Hub-height axial velocity was extracted from the simulation results at the rotor plane $(x=0 d)$ and at the point of static pressure equalisation across the flow, which occurred approximately $2 d$ downstream of the rotor plane. Figure A.10 shows the $\kappa=\left(1-a_{2 i}\right) /\left(1-a_{4 i}\right)$ ratios in the three blockage ratios at three tip speed ratios $(\lambda=5.00,6.00,7.00)$. Generally, $\kappa$ increases with $\lambda$, which corresponds to greater momentum removal from the flow. The $\kappa$ ratio is larger for the blocked than the unblocked rotor for a given $(B, \lambda)$ combination, as the greater blade solidity of the blocked rotor results in higher rotor thrust. Increasing the blockage ratio reduces the $\kappa$ ratio, as the effect of flow confinement is to constrain the wake expansion, resulting in higher downstream velocities and thus smaller $\kappa$ ratios. The $\kappa$ ratio is generally constant (within 5-10\%) across the majority of the blade span, decreasing at $r / R>0.8$ towards the blade tips as tip loss effects become significant. Flow acceleration around the nacelle is observed to affect the $\kappa$ ratio at small spanwise distances $r / R<0.3$.

[1] Bahaj, A. S., Batten, W. M. J. and McCann, G. [2007], 'Experimental verifications of numerical predictions for the hydrodynamic performance of horizontal axis marine current turbines', Renewable Energy 32, 2479-2490.

[2] Bai, G., Li, J., Fan, P. and Li, G. [2013], 'Numerical investigations of the effects of different arrays on power extractions of horizontal axis tidal current turbines', Renewable Energy 53, 180-186.

[3] Betz, A. and Prandtl, L. [1919], 'Schraubenpropeller mit geringstem Energieverlust', Nachrichten von der Gesellschaft der Wissenschaften zu Göttingen pp. 193-217.

[4] Buhl Jr, M. L. [2005], A new empirical relationship between thrust coefficient and induction factor for the turbulent windmill state, Technical Report NREL/TP-500-36834, National Renewable Energy Laboratory. 

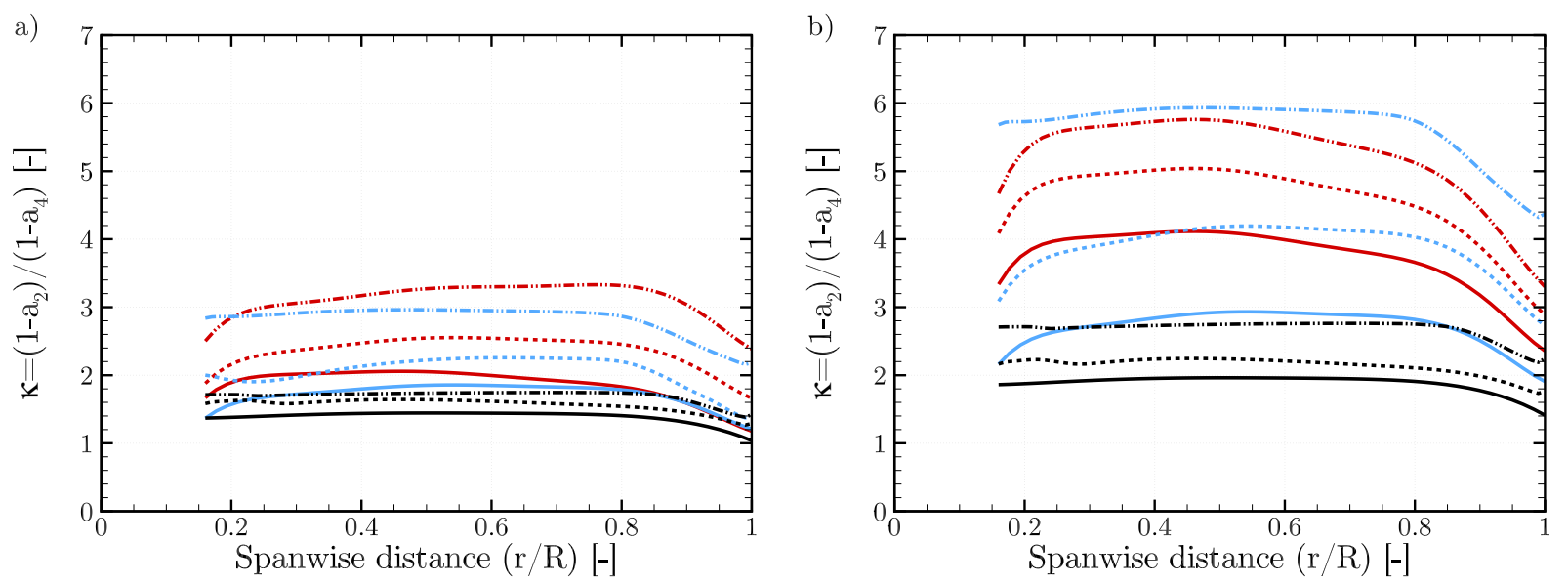

Figure A.10: $\kappa=\left(1-a_{2 i}\right) /\left(1-a_{4 i}\right)$ ratio for a single rotor of the unblocked (a) and blocked (b) rotor designs of Wimshurst and Willden [30] operating in unconfined (red), moderately confined ( $B=0.507$, blue), and significantly confined $(B=0.1960$, black) conditions. Three different tip speed ratios $\lambda$ are shown in each blockage case; $\lambda=5.00$ (solid), $\lambda=6.00$ (dashed), and $\lambda=7.00$ (dot-dashed).

[5] Burton, T., Sharpe, D., Jenkins, N. and Bossanyi, E. [2001], Wind Energy Handbook, West Sussex: John Wiley \& Sons, Ltd.

[6] Chamorro, L. P., Troolin, D. R., Lee, S.-J., Arndt, R. E. A. and Sotiropoulos, F. [2013], 'Threedimensional flow visualization in the wake of a minature axial-flow hydrokinetic turbine', Experiments in Fluids 54(2), 1459.

[7] Edmunds, M., Malki, R., Williams, A. J., Masters, I. and Croft, T. N. [2014], 'Aspects of tidal stream turbine modelling in the natural environment using a coupled BEM-CFD model', International Journal of Marine Energy 7, 20-42.

[8] Fuglsang, P. and Bak, C. [2004], 'Development of the Risø Wind Turbine Airfoils', Wind Energy 7, 145162.

[9] Garrett, C. and Cummins, P. [2007], 'The efficiency of a turbine in a tidal channel', Journal of Fluid Mechanics 588, 243-251.

[10] Glauert, H. [1935], Aerodynamic theory, in 'Airplane propellers', Julius Springer: Berlin, pp. 169-360.

[11] Houlsby, G. T. and Vogel, C. R. [2016], 'The power available to tidal turbines in an open channel flow', ICE Energy 169, 1-10.

[12] Kramer, S. C. and Piggott, M. D. [2016], 'A correction to the enhanced bottom drag parameterisation of tidal turbines', Renewable Energy 92, 385-396.

[13] Masters, I., Chapman, J. C., Willis, M. R. and Orme, J. A. C. [2011], 'A robust blade element momentum theory model for tidal stream turbines including tip and hub loss corrections', Journal of Marine Engineering and Technology 10(1), 25-35.

[14] McNaughton, J. [2013], 'Turbulence modelling in the near-field of an axial flow tidal turbine using Code Saturne', PhD thesis, University of Manchester.

[15] Menter, F. R. [1994], 'Two-equation eddy-viscosity turbulence models for engineering applications', AIAA Journal 32(8), 1598-1605.

[16] Moriarty, P. [2008], 'Database for validation of design load extrapolation techniques', Wind Energy 11, $559-576$. 
[17] Nishino, T. and Willden, R. H. J. [2013], 'Two-scale dynamics of flow past a partial cross-stream array of tidal turbines', Journal of Fluid Mechanics 730, 220-244.

[18] Ouro, P., Harrold, M., Stoesser, T. and Bromley, P. [2017], 'Hydrodynamic loadings on a horizontal axis tidal turbine prototype', Journal of Fluids and Structures 71, 78-95.

[19] Sarlak, H., Nishino, T., Martínez-Tossas, L. A., Meneveau, C. and Sørensen, J. N. [2016], 'Assessment of blockage effects on the wake characteristics and power of wind turbines', Renewable Energy 93, 340-352.

[20] Schluntz, J. and Willden, R. H. J. [2013], The effect of rotor design on the power output of closely packed tidal turbines, in 'Tenth European Wave and Tidal Energy Conference', Aalborg, Denmark.

[21] Schluntz, J. and Willden, R. H. J. [2015], 'The effect of blockage on tidal turbine rotor design and performance', Renewable Energy 81, 432-441.

[22] Shen, W. Z., Sørensen, J. N. and Mikkelsen, R. [2005], 'Tip loss correction for actuator/Navier-Stokes computations', Journal of Solar Energy Engineering 127, 209-213.

[23] Sun, Z., Chen, J., Shen, W. Z. and Zhu, W. J. [2016], 'Improved blade element momentum theory for wind turbine aerodynamic computations', Renewable Energy 96, 824-831.

[24] Tangler, J. L. [1982], 'Comparison of wind turbine performance prediction and measurement', Journal of Solar Energy Engineering 104, 84-88.

[25] Vaz, J. R. P., Pinho, J. T. and Mesquita, A. L. A. [2011], 'An extension of BEM method applied to horizontal-axis wind turbine design', Renewable Energy 36, 1734-1740.

[26] Vogel, C. R. and Willden, R. H. J. [2017], 'Multi-rotor tidal stream turbine fence performance and operation', International Journal of Marine Energy 19, 198-206.

[27] Vogel, C. R., Willden, R. H. J. and Houlsby, G. T. [2016], 'Effect of free surface deformation on the extractable power of a finite width turbine array', Renewable Energy 88, 317-324.

[28] Whelan, J. I., Graham, J. M. R. and Pieró, J. [2009], 'A free surface and blockage correction for tidal turbines', Journal of Fluid Mechanics 624, 281-291.

[29] Wimshurst, A., Vogel, C. R. and Willden, R. H. J. [2018], 'Cavitation limits on tidal turbine performance', Ocean Engineering 152, 223-233.

[30] Wimshurst, A. and Willden, R. H. J. [2016], Computational analysis of blockage designed tidal turbine rotors, in 'Second International Conference on Renewable Energies Offshore', Lisbon, Portugal.

[31] Wimshurst, A. and Willden, R. H. J. [2017], 'Analysis of a tip correction factor for horizontal axis turbines', Wind Energy 20, 1515-1528.

[32] Wimshurst, A. and Willden, R. H. J. [2018], 'Computational observations of the tip loss mechanism experienced by horizontal axis rotors', Wind Energy 21, 544-557. 